\title{
Mechanism and Kinetics of Stabilization Reaction of Polyacrylonitrile and Related Copolymers II. Relationships between Isothermal DSC Thermograms and FT-IR Spectral Changes of Polyacrylonitrile in Comparison with the Case of Acrylonitrile/Methacrylic Acid Copolymer
}

\author{
Hideto $\mathrm{KAKIDA}^{\dagger}$ and Kohji TASHIRO* \\ Central Technology Research Laboratories, Mitsubishi Rayon Co., Ltd., \\ Otake, Hiroshima 739-06, Japan \\ * Department of Macromolecular Science, Graduate School of Science, Osaka University, \\ Toyonaka, Osaka 560, Japan \\ (Received October 9, 1996)
}

\begin{abstract}
The relationship between thermal behavior and structural change has been studied by an organized combination of isothermal DSC thermograms and FT-IR spectra measured for the stabilization reaction of polyacrylonitrile (PAN) in comparison with the case of acrylonitrile/methacrylic acid (AN/MAA) copolymer. The isothermal DSC exothermic thermogram of PAN is much broader and the structural changes proceed much more slowly than the case of AN/MAA copolymer. On the stabilization of PAN, at first, some nitrile groups change into amide groups, which initiate the dehydrogenation and cyclization reactions. This dehydrogenation reaction proceeds rather faster than the cyclization. The comonomer MAA was considered to accelerate the dehydrogenation reaction more effectively than the cyclization reaction.
\end{abstract}

KEY WORDS Polyacrylonitrile / Acrylonitrile-Methacrylic Acid Copolymer / Carbon Fiber /

Stabilization / Differential Scanning Calorimetry / Fourier-Transform-Infrared Spectra /

Polyacrylonitriles (PAN) containing acidic comonomers are the most commonly used precursors for carbon fibers because of the excellent performance of PAN-based carbon fibers. ${ }^{1}$

Production process of PAN-based carbon fibers involves the stabilization of oriented acrylic fibers and the carbonization of them at an elevated temperature. ${ }^{2}$ Stabilization is the most important process which converts acrylic fibers to infusible and nonflammable fibers by heating at $200-300^{\circ} \mathrm{C}$ for about one hour in an oxidative atmosphere. The thus stabilized fibers can be heated up to the carbonization temperature (1000$2000^{\circ} \mathrm{C}$ ) in an inert atmosphere. ${ }^{2}$

To accelerate the stabilization reaction, several comonomers such as methacrylic acid (MAA), acrylic acid (AA), methacrylate (MA), and acrylamide (AAM) are often copolymerized into PAN. But, the role of the comonomers on the kinetics of the stabilization reaction and the mechanism of the stabilization reaction itself are not so clear yet now. ${ }^{2}$

In order to solve these problems of the stabilization of PAN and related copolymers, we have developed a new technical idea to combine the experimental data of isothermal DSC thermograms with FT-IR spectra. In the previous paper, ${ }^{3}$ we revealed the intimate relationship between the thermal behavior and the structure changes by such an organized combination of isothermal DSC thermograms and FT-IR spectra measured for the stabilization reaction of an AN/MAA copolymer, and also found for the first time the induction period of the cyclic structure formation.

After that work, ${ }^{3}$ the isothermal DSC thermograms of PAN homopolymer were found to be very different from those of an AN/MAA copolymer. The isothermal DSC thermogram of PAN shows a sharp but "small" exothermic peak at an early stage of the reaction, followed by a broad and "large" exothermic peak. This behavior is quite contrast to the case of the AN/MAA copolymer, in which almost only a sharp and "large" exothermic peak can be observed at an early stage.

The present study has therefore been undertaken so as to clarify the difference of stabilization mechanisms between PAN and the AN/MAA copolymer through our new method combining isothermal DSC thermograms and FT-IR spectra.

\section{EXPERIMENTAL}

\section{Materials}

PAN and the AN/MAA copolymer (CP1) were synthesized by an aqueous suspension polymerization method with redox initiator. The composition of AN/MAA copolymer was $\mathrm{AN}: \mathrm{MAA}=98.5: 1.5$ in weight ratio in feed. The weight-averaged molecular weights of these polymers were about $2 \times 10^{5}$. The obtained two kined of polymers showed the same triad tacticity as $m m=$ $0.27, m r=0.50$, and $r r=0.23$ when evaluated by means of ${ }^{13} \mathrm{C}-\mathrm{NMR}$.

The polymer powders filtered through a 400 mesh screen were supplied to the DSC and FT-IR measurements.

\section{DSC and FT-IR Measurements}

The isothermal DSC and FT-IR measurements were carried out on the basis of the methods described previously. ${ }^{3}$ The infrared spectra were measured at room temperature for the samples quenched from the various points of the DSC thermograms as seen in Figure 5, for an example.

\footnotetext{
† To whom correspondence should be addressed.
} 


\section{RESULTS AND DISCUSSION}

\section{Isothermal DSC Measurements}

Figure 1 shows the isothermal DSC thermograms of the PAN and the CP1 measured at $240^{\circ} \mathrm{C}$ under air flow. The scales of the ordinate are different between these two samples; the thermogram of the PAN is expanded longitudinally about 100 times larger than that of the CP1. The isothermal DSC thermograms of the PAN are very different from those of the CP1. The thermograms of the CP1 show apparently one sharp exothermic peak only at the early time region. On the other hand, the thermogram of the PAN consists of a small peak appearing at an early period and a broad peak at a later period, i.e., the stabilization reactions of the PAN seem to be composed of the rapid and slow exothermic processes, while that of the CP1 contains only the rapid exothermic processes.

In Figure 2, the isothermal DSC thermogram and the total amount of heat evolved during the stabilization process are compared between the PAN and the CP1. The total amount of evolved heat was evaluated by drawing the base line horizontally, as indicated by a broken line, at the heat flow level of the induction period. The existence of the induction period in the stabilization reaction was found for the first time as described in the previous report. ${ }^{3}$ The ordinate of Figure 2(a), is about 200 times largely expanded compared with that of Figure 2(b). It can be seen that the rate of heat evolution during the stabilization of the PAN is much lower than that of the CP1. It is also noteworthy that almost the same amount of heat, 3290 and $3260 \mathrm{~J} \mathrm{~g}^{-1}$, are evolved for the PAN and the CP1 during the stabilization process, respectively, although the reaction behavior and the rate of reaction are much different from each other. Comparing Figure 1 with Figure 2, the comonomer MAA is considered to play an important role in accelerating the slow exothermic process of the PAN homopolymer without any change in the amount of heat evolution.

Figure 3 shows the isothermal DSC thermograms measured at various temperatures under air flow for the PAN homopolymer. In the early regions of these thermograms there are nearly flat region and the period of the region becomes shorter as the temperature is

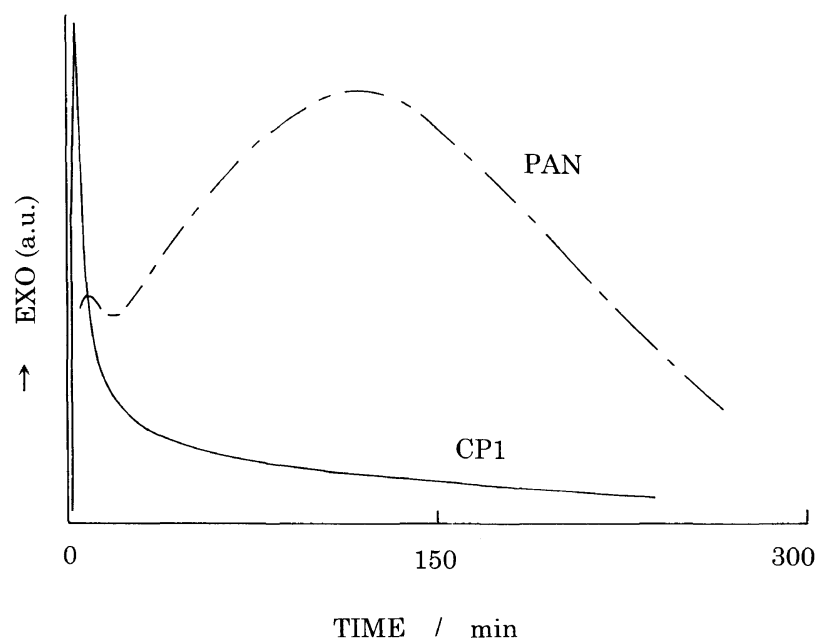

Figure 1. Isothermal DSC thermograms of PAN and AN/MAA copolymer measured at $240^{\circ} \mathrm{C}$ under air flow. increased. These nearly flat regions should be considered as the induction periods of the stabilization reactions, and were observed for the CP1 as reported in a previous paper. ${ }^{3}$ In Figure 4 , the Arrhenius plot is made for the induction periods obtained at $215,230,250$, and $260^{\circ} \mathrm{C}$, where the induction period $t_{\mathrm{i}}$ is defined as the standing point of the thermal evolution (see Figure 3). From this plot an activation energy was evaluated to be $c a .22$ $\mathrm{kcal} \mathrm{mol}^{-1}$. This value is about the same with that of the CP1.

\section{Structural Changes during Exothermic Reactions}

The molecular structural changes of the PAN homopolymer which occur during the isothermal heat evolution process in DSC were investigated by means of
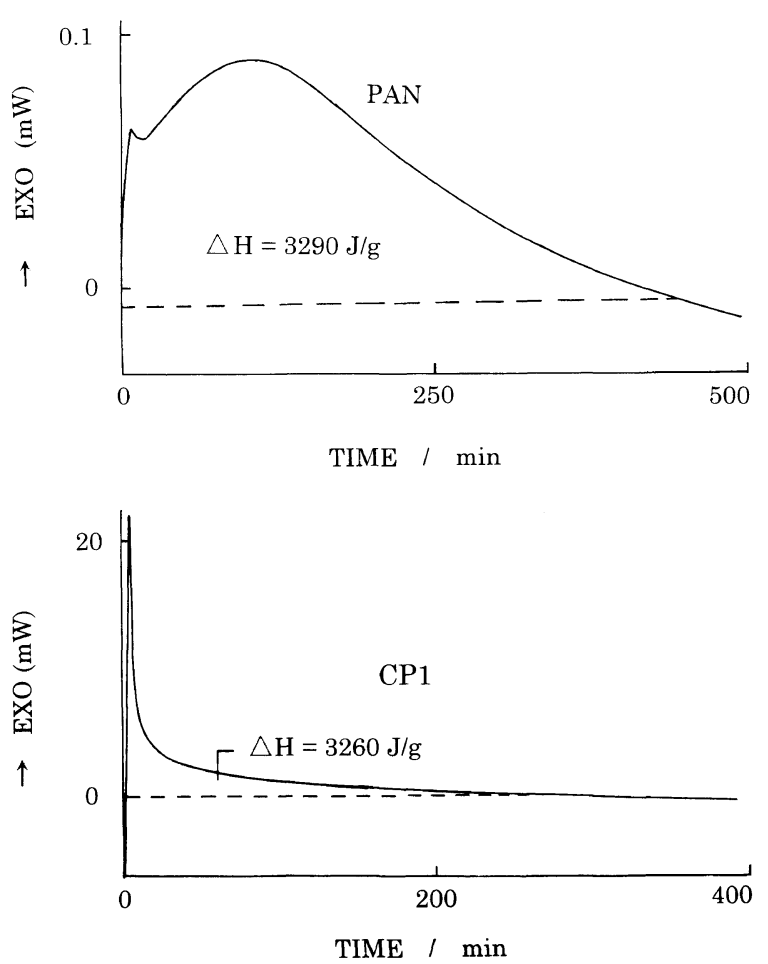

Figure 2. Isothermal DSC thermograms and total amount of heat evolved during stabilization at $240^{\circ} \mathrm{C}$ for PAN and AN/MAA copolymer.

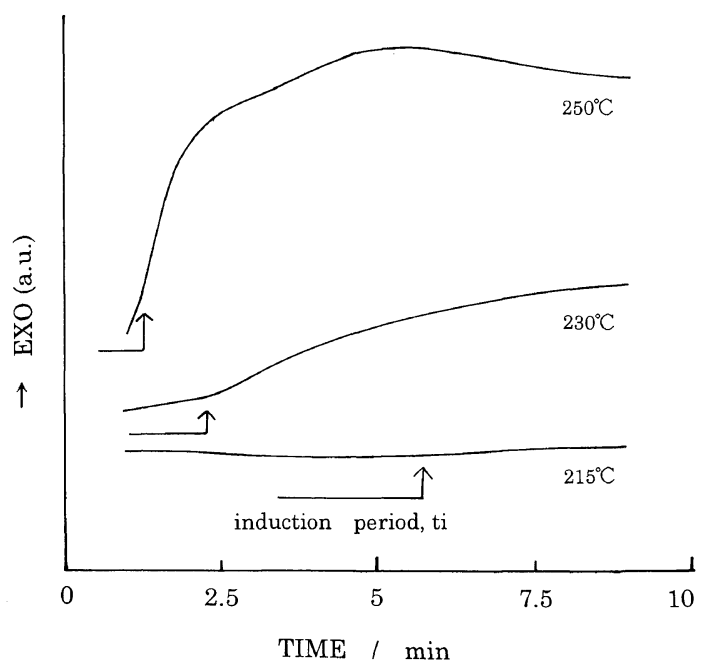

Figure 3. Isothermal DSC thermograms of PAN measured at several temperatures under air flow. 


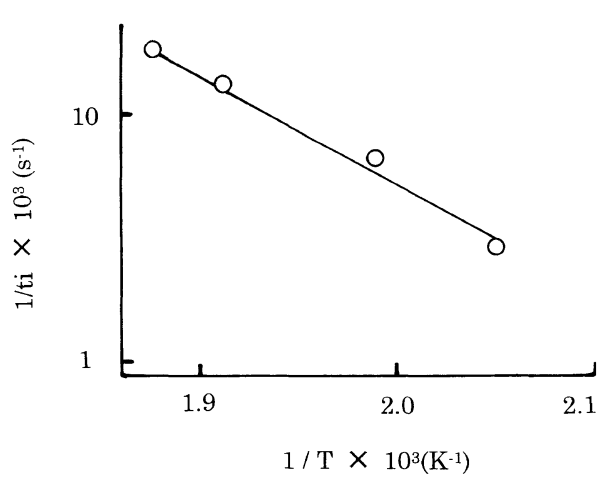

Figure 4. The Arrhenius plot of the induction period $\left(t_{\mathrm{i}}\right)$ measured at $215,230,250$, and $260^{\circ} \mathrm{C}$ for PAN.

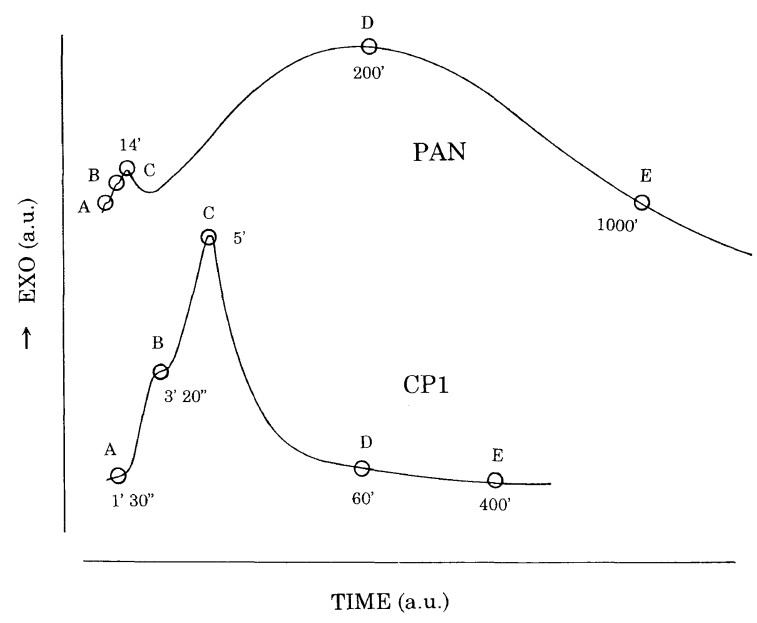

Figure 5. The schematic DSC thermograms of PAN and AN/MAA copolymer measured at $230^{\circ} \mathrm{C}$ under air. At the several points indicated by marks the IR spectra were observed as shown in Figures 6 and 7 .

FT-IR. In Figure 5, a typical isothermal DSC thermogram of the PAN homopolymer is illustrated schematically in comparison with that of the CP1. The exothermic phenomena of the PAN are apparently more complicated than those of the CP1. The thermogram of the PAN is composed of several stages; an induction period A, an exothermic shoulder $\mathrm{B}$, a small peak $\mathrm{C}$ and an exothermic large broad peak D as indicated in this figure. The broad peak is absent in the thermogram of the CP1 (see Figure 1). The IR spectra were measured for the PAN at several points indicated by open circles in Figure 5 in order to get the preliminary information for the structural changes occurring during the isothermal exothermic DSC thermograms and were compared with the spectra of the CP1 reported in the previous paper. ${ }^{3}$ The IR spectra are shown in Figure 6 for the PAN and in Figure 7 for the CP1. It can be seen for both the PAN and the CP1 that the bands at 2240 and $1450 \mathrm{~cm}^{-1}$ decrease in intensity while the bands at 1600 and 810 $\mathrm{cm}^{-1}$ increase the intensities as the retention time in DSC becomes longer. Unexpectedly, however, when Figure 6 is compared with Figure 7, the spectrum at the small peak position $\mathrm{C}$ of the DSC thermogram of the PAN is almost the same with that at the point $C$ of the $\mathrm{CP} 1$, and the spectrum at main peak position $\mathrm{D}$ of the PAN is very similar to that of the CP1 measured at point $\mathrm{D}$ of 60 minutes, which is the position at which the exothermic heat flow is slowing down.

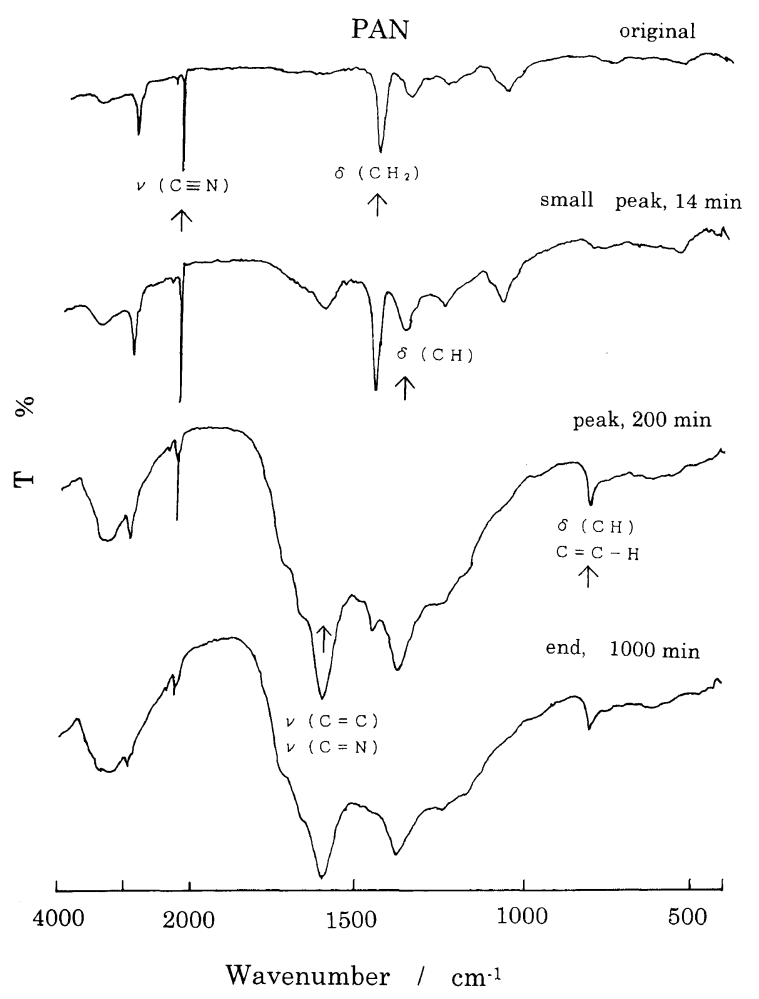

Figure 6. The IR spectra of PAN which were observed at the points indicated by $\bigcirc$ marks shown in Figure 5 .

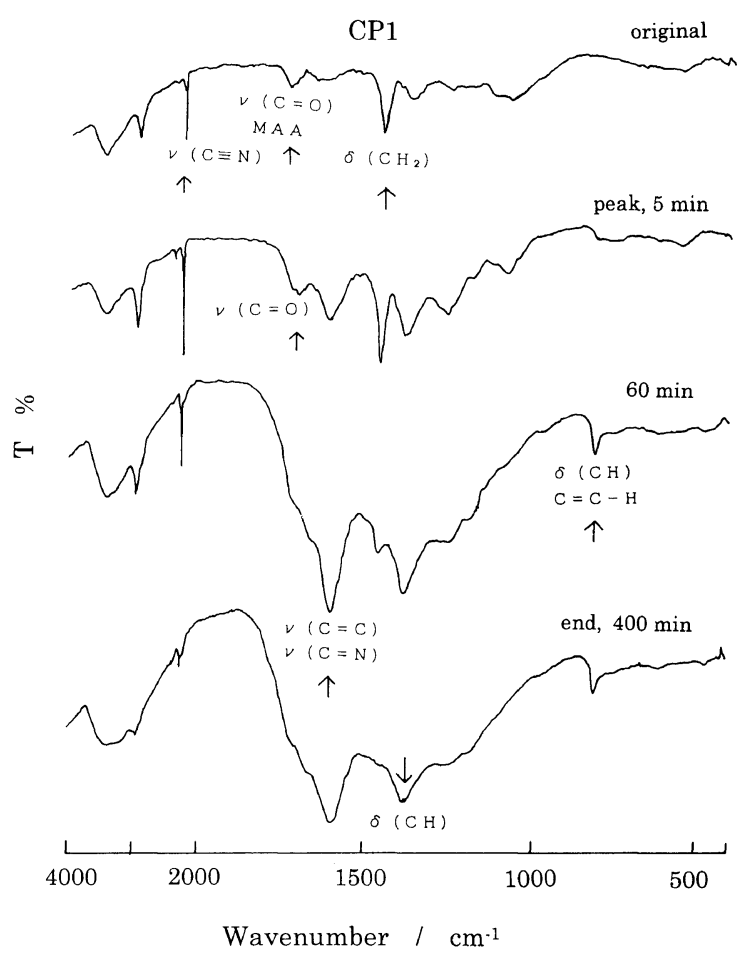

Figure 7. The IR spectra of AN/MAA copolymer which were observed at the points indicated by $\bigcirc$ marks shown in Figure 5 .

In order to clarify the difference in the structure changes between the PAN and the CP1, the comparison was made between the isothermal DSC thermograms and the intensity changes of the several main IR bands in the same time scale in Figure 8. As described in the previous paper, ${ }^{3}$ in the case of the CP1, the IR band intensities change drastically in the remarkable exothermic region for the early 50 minutes; the bands at 1600 and $810 \mathrm{~cm}^{-1}$ 

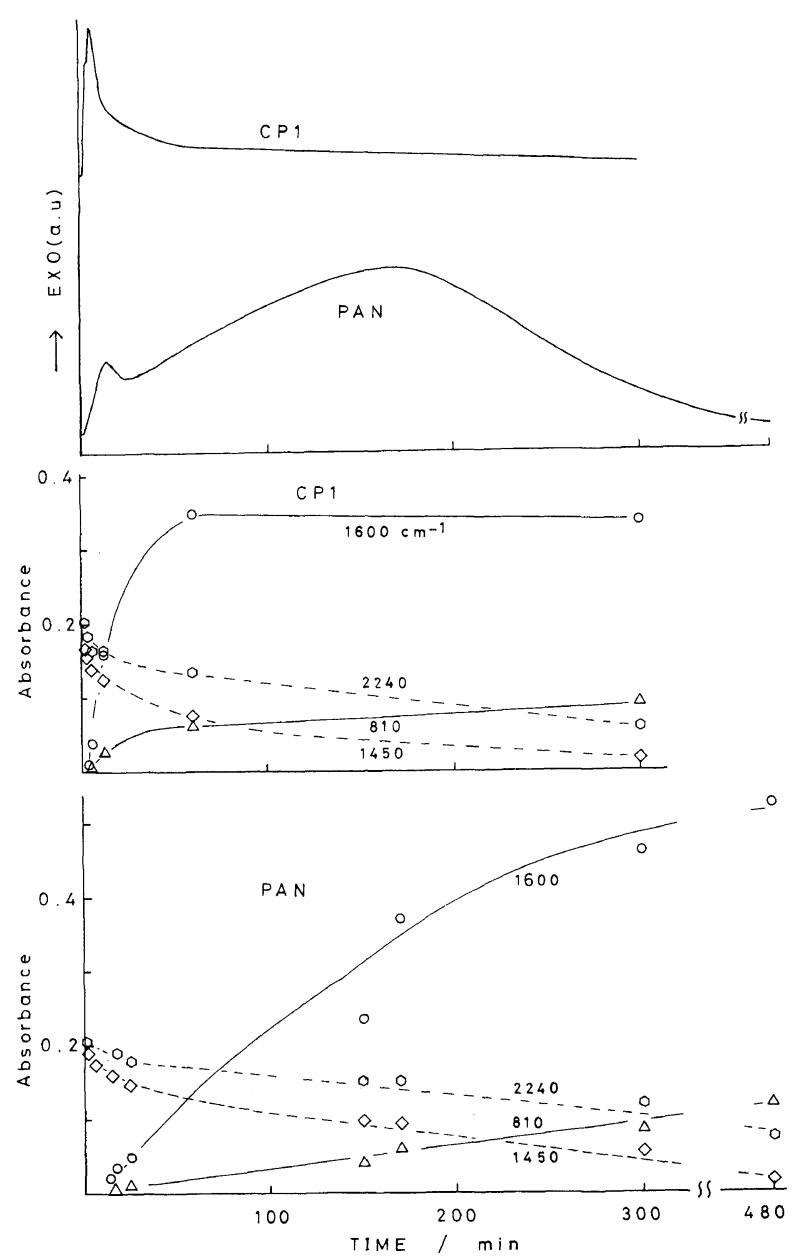

Figure 8. The comparisons of the isothermal DSC thermograms and the intensity changes of several main IR bands between PAN and AN/MAA copolymer.

increase the intensities while the bands at 2240 and $1450 \mathrm{~cm}^{-1}$ decrease. On the other hand, in the case of the PAN, the intensities of these IR bands change very slowly and only the $1450 \mathrm{~cm}^{-1}$ band intensity changes somewhat steeply than the others during the early period. From these data, it is clear that the MAA comonomer accelerates the dehydrogenation and cyclization reactions, and it may be assumed that the small exothermic peak at early region of the PAN occurs mainly due to the dehydrogenation reaction.

In order to get the details of the initiation $(0-10 \mathrm{~min})$ stage of stabilization reaction of the PAN, structural changes during the induction period were studied by FT-IR. In Figure 9, the changes of the IR spectra during the induction period are compared between the PAN and the CP1 for the samples which were annealed in DSC cell at $200^{\circ} \mathrm{C}$ under air flow. In the case of the $\mathrm{CP} 1,{ }^{3}$ the $1740 \mathrm{~cm}^{-1}$ band decreases and the $1700 \mathrm{~cm}^{-1}$ band appears and increases in intensity, indicating that some MAA units may react with the adjacent AN units in this time region. On the other hand, for the PAN, the 1680 $\mathrm{cm}^{-1}$ band appears and increases in intensity, suggesting that the amide groups are formed during the induction period. That is to say, the stabilization reaction of the PAN may be initiated through the formation of some amide groups from nitrile groups. In Figure 10, the isothermal DSC thermogram measured at the early stage

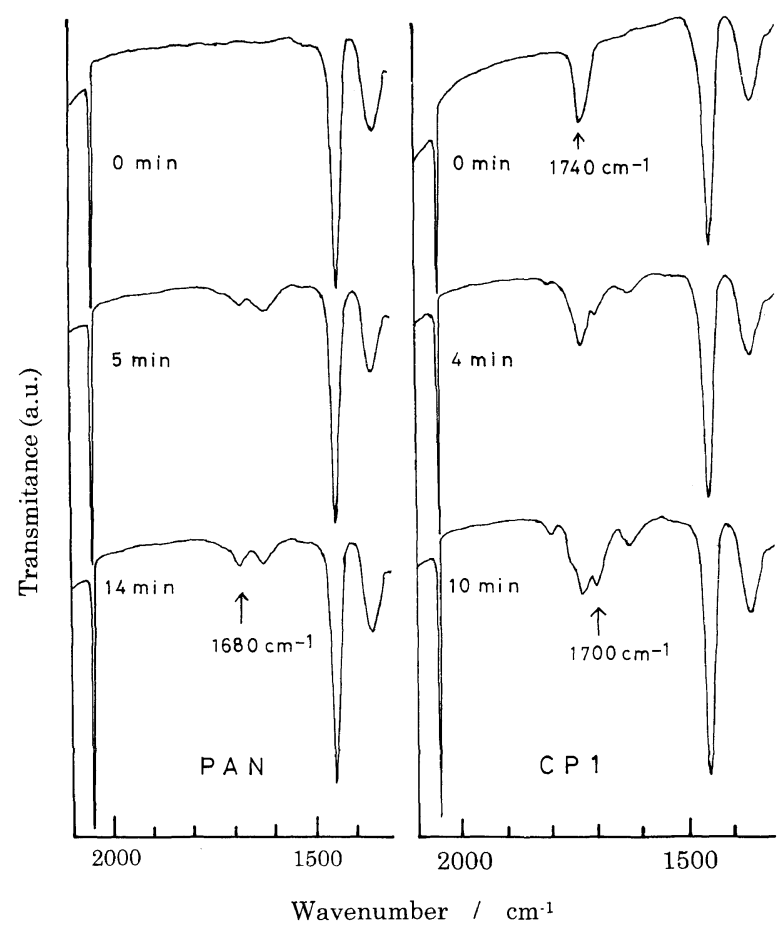

Figure 9. The changes of IR spectra during induction periods of PAN and AN/MAA copolymers.

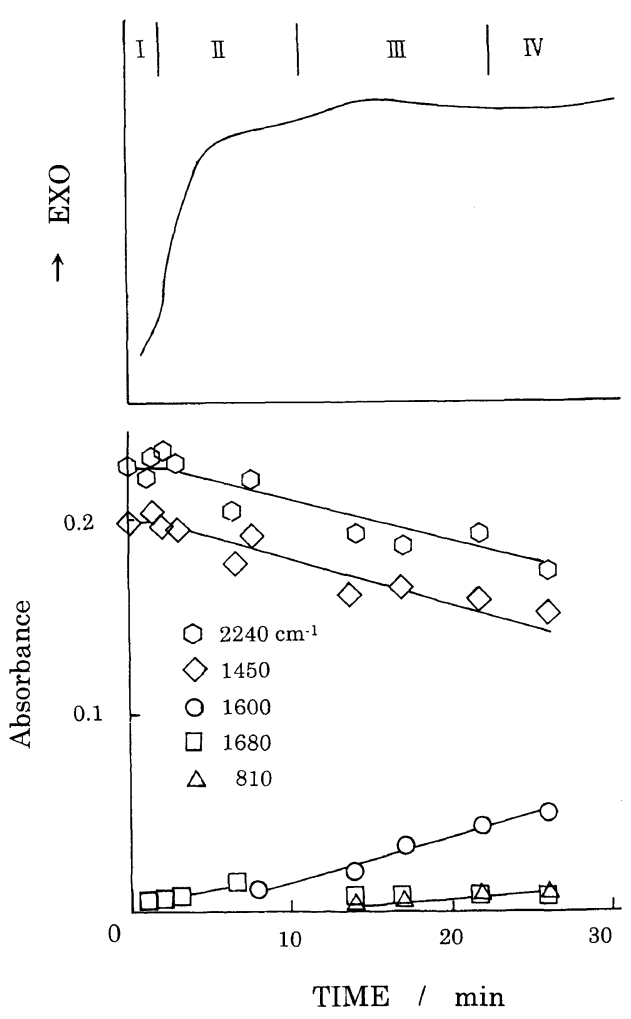

Figure 10. The early region of the isothermal DSC thermogram measured at $230^{\circ} \mathrm{C}$ and the corresponding changes of the intensity of the several main IR bands.

at $230^{\circ} \mathrm{C}$ in air $(0-30 \mathrm{~min})$ is shown together with the corresponding time dependence of the IR band intensity changes. In the induction region $[\mathrm{I}]$, the 1620 and $1680 \mathrm{~cm}^{-1}$ bands begin to appear and increase their intensities with time, but the 2240 and $1450 \mathrm{~cm}^{-1}$ bands show almost no intensity change. In the region [II] or the exothermic shoulder region, the 2240 and $1450 \mathrm{~cm}^{-1}$ 
Table I. Principal IR bands in solid and degraded PAN and AN/MAA copolymer structures

\begin{tabular}{lc}
\hline \multicolumn{1}{c}{ Functional groups } & Wavenumber $/ \mathrm{cm}^{-1}$ \\
\hline $\mathrm{NH}_{2}$ & 3390,3356 \\
$\mathrm{NH}$ & 3230 \\
$\mathrm{CH}_{2}$ & 2945,2920 \\
$\mathrm{CH}$ & 2895 \\
$\mathrm{C} \equiv \mathrm{N}$ (saturated) & 2240 \\
$\mathrm{C}=\mathrm{O}$ (unreacted MAA) & 1740 \\
$\mathrm{C}=\mathrm{O}$ (reacted MAA) & 1700 \\
$\mathrm{C}=\mathrm{O}$ (amide) & 1680 \\
$\mathrm{~N}-\mathrm{H}$ (amide) & 1620 \\
$\mathrm{C}=\mathrm{N}, \mathrm{C}=\mathrm{C}$ mixed & $1620^{\mathrm{a}}$ \\
$\mathrm{C}=\mathrm{N}, \mathrm{C}=\mathrm{C}, \mathrm{N}-\mathrm{H}$ mixed & $1580^{\mathrm{a}}$ \\
$\mathrm{CH}$ & 1450 \\
$\mathrm{CH}, \mathrm{NH}$ & 1380 \\
$\mathrm{C}-\mathrm{C}, \mathrm{C}-\mathrm{N}$ mixed & 1250 \\
$\mathrm{C}=\mathrm{C}-\mathrm{H}$ & 810 \\
\hline
\end{tabular}

a These bands are often not resolved and appear at $1600 \pm 10$ $\mathrm{cm}^{-1}$.

bands begin to decrease their intensities. The $1600 \mathrm{~cm}^{-1}$ band begins to appear in this region. In the small peak region [III], the $810 \mathrm{~cm}^{-1}$ band appears and the 1600 $\mathrm{cm}^{-1}$ band increases in intensity. In the region [IV], the rate of heat evolution increases very slowly toward main broad exothermic peak, just when the 2240 and 1450 $\mathrm{cm}^{-1}$ bands continue to decrease and the 1600 and 810 $\mathrm{cm}^{-1}$ bands increase their intensities.

Table I summarizes the IR band assignments for the thermally degraded the PAN and the CP1 with reference to Fochler et al. ${ }^{4}$ and Sivy et al. ${ }^{5}$

On the stabilization of the PAN, at first, a little nitrile groups change into amide groups as shown in Figure 11(a), and then the dehydrogenation of backbone and cyclization of nitrile groups are initiated by amide groups (Figures 11(b), (c)). Among these reactions the dehydrogenation is more predominant than the cyclization as seen from the different rate of the infrared intensity change between the $1450 \mathrm{~cm}^{-1} \mathrm{CH}_{2}$ bending band and the $2240 \mathrm{~cm}^{-1} \mathrm{C} \equiv \mathrm{N}$ stretching band. The amount of heat evolution due to dehydrogenation is $243 \mathrm{k} \mathrm{J} \mathrm{mol}^{-1}$ which is larger than the $59 \mathrm{k} \mathrm{J} \mathrm{mol}^{-1}$ of cyclization. ${ }^{1}$ So, the experimental finding that the dehydrogenation is

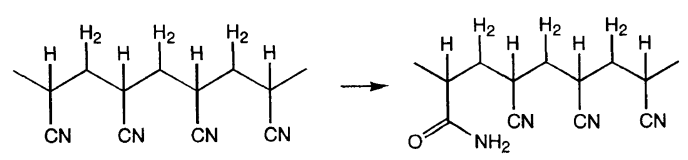

(a)

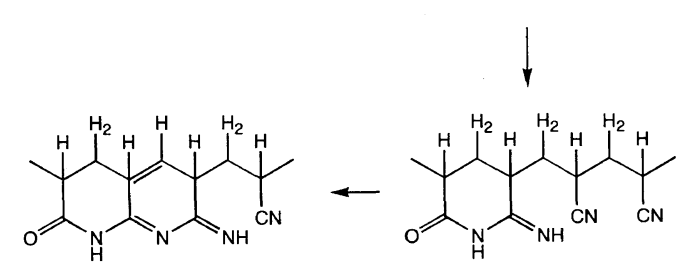

(c)

(b)

Figure 11. The chemical structure changes which occur in the early stage of the stabilization of PAN.

more predominant than the cyclization seems to be a reason why the small exothermic peak appears in this region. After this small peak, the dehydrogenation and the cyclization proceed at the same but very slow pace and therefore the broad exothermic main peak appears in the DSC thermogram. While, on the stabilization of the CP1 which contains MAA as comonomer, some MAA comonomers react with the adjacent nitrile groups, which accelerate the dehydrogenation and cyclization reaction much faster than the stabilization of the PAN. The dehydrogenation proceeds rather faster and slowdowns in an earlier stage than the cyclization. The comonomer MAA seems to accelerate the dehydrogenation and also cyclization, and the acceleration by MAA is more effective on the dehydrogenation than the cyclization.

\section{REFERENCES}

1. A. Gupta, D. K. Paliwal, and P. Bajaj, J. Macromol. Sci. C, 31, 1 (1991).

2. J. B. Donnet and R. C. Bansal, "Carbon Fiber," 2nd ed, Marcel Dekker, New York, N. Y., 1990.

3. H. Kakida, K. Tashiro, and M. Kobayashi, Polym. J., 28, 30 (1996).

4. H. S. Fochler, J. R. Mooney, L. E. Ball, R. D. Boyer, and J. G. Grasseli, Spectrochim. Acta, 41A, 271 (1985).

5. G. T. Sivy and M. M. Coleman, Carbon, 19, 127 (1981). 